\title{
Multistep Bloch-line-mediated Walker breakdown in ferromagnetic strips
}

\section{Hutner, Johanna}

2019-05-24

Hutner , J , Herranen , T \& Laurson , L 2019 , ' Multistep Bloch-line-mediated Walker breakdown in ferromagnetic strips ' , Physical Review B , vol. 99 , no. 17 , 174427 . https://doi.org/10.1103/PhysRev

http://hdl.handle.net/10138/320808

https://doi.org/10.1103/PhysRevB.99.174427

publishedVersion

Downloaded from Helda, University of Helsinki institutional repository.

This is an electronic reprint of the original article.

This reprint may differ from the original in pagination and typographic detail.

Please cite the original version. 


\title{
Multistep Bloch-line-mediated Walker breakdown in ferromagnetic strips
}

\author{
Johanna Hütner, ${ }^{1,2}$ Touko Herranen, ${ }^{1}$ and Lasse Laurson ${ }^{1,3, *}$ \\ ${ }^{1}$ Helsinki Institute of Physics and Department of Applied Physics, Aalto University, P.O. Box 11100, FI-00076 Aalto, Espoo, Finland \\ ${ }^{2}$ Aalto Science Institute, Aalto University, P.O. Box 11100, FI-00076 Aalto, Espoo, Finland \\ ${ }^{3}$ Computational Physics Laboratory, Tampere University, P.O. Box 692, FI-33014 Tampere, Finland
}

(Received 26 November 2018; revised manuscript received 28 January 2019; published 24 May 2019)

\begin{abstract}
A well-known feature of magnetic field driven dynamics of domain walls in ferromagnets is the existence of a threshold driving force at which the internal magnetization of the domain wall starts to precess-a phenomenon known as the Walker breakdown-resulting in an abrupt drop of the domain-wall propagation velocity. Here, we report on micromagnetic simulations of magnetic field driven domain-wall dynamics in thin ferromagnetic strips with perpendicular magnetic anisotropy which demonstrate that in wide enough strips Walker breakdown is a multistep process: It consists of several distinct velocity drops separated by short linear parts of the velocity vs field curve. These features originate from the repeated nucleation, propagation, and annihilation of an increasing number of Bloch lines within the domain wall as the driving field magnitude is increased. This mechanism arises due to magnetostatic effects breaking the symmetry between the two ends of the domain wall.
\end{abstract}

DOI: 10.1103/PhysRevB.99.174427

\section{INTRODUCTION}

Domain-wall (DW) dynamics driven by applied magnetic fields [1-3] or spin-polarized electric currents [4-6] is an active field of research catalyzed by both fundamental physics interests as well as promising applications in technology. One of the most striking features of DW dynamics is that one typically observes a nonmonotonic driving force dependence of the DW propagation velocity $v_{\mathrm{DW}}$. Considering field-driven DW dynamics, for small applied fields $B_{\text {ext }}$, $v_{\text {DW }}$ first increases with $B_{\text {ext }}$, followed by a sudden drop of $v_{\text {Dw }}$. The latter originates from an instability known as the Walker breakdown [3], where the internal DW magnetization starts precessing at $B_{\text {ext }}=B_{\mathrm{W}}$, with $B_{\mathrm{W}}$ known as the Walker field. This leads to a reduced $v_{\mathrm{DW}}$ for $B_{\text {ext }}>B_{\mathrm{W}}$ as part of the energy of the driving field is dissipated by the precessional magnetization dynamics within the DW.

The widely used one-dimensional (1D) models [7] describe this precession by a single angular variable, and have been demonstrated to successfully capture the DW dynamics in nanowire geometries [8]. However, this simple description fails in wide enough strips. In such systems an instability analogous to the Walker breakdown in nanowires is known to proceed in a spatially nonuniform fashion via repeated nucleation and propagation of Bloch lines (BLs) within the DW [9-11]. BLs are topologically stable magnetization textures corresponding to localized transition regions separating different chiralities of the Bloch DW. In the case of thin strips considered here, BLs are lines threading the strip in the thickness direction, and are hence referred to as vertical Bloch lines (VBLs) [11,12]. Even if the study of BLs especially in the context of bubble materials has a long history dating back to the 1970s $[13,14]$, the various BL excitation

*lasse.laurson@tuni.fi modes responsible for the velocity drop in strips of different geometries remain to be understood.

Hence, we perform here extensive micromagnetic simulations of field-driven DW dynamics considering thin $\mathrm{CoPtCr}$ strips with strong perpendicular magnetic anisotropy as example systems (see Fig. 1). We study in detail the dependence of the DW propagation velocity $v_{\mathrm{DW}}$ on the applied field $B_{\text {ext }}$, as well as the onset of precessional dynamics at $B_{\mathrm{ext}}=B_{\mathrm{W}}$ for a wide range of strip widths $L_{y}$. Remarkably, by carefully inspecting the "fine structure" of the Walker breakdown, we find that for wide enough strips the large velocity drop in the $v_{\mathrm{DW}}\left(B_{\text {ext }}\right)$ curve observed previously [9] actually consists of several distinct, smaller velocity drops, separated by short linearly increasing parts of $v_{\mathrm{DW}}\left(B_{\text {ext }}\right)$. Our analysis of the corresponding VBL dynamics within the DW shows that this behavior arises due to a sequence of distinct excitations of the DW magnetization. Thereby, the number of VBLs present within the DW increases with $B_{\text {ext }}$ in discrete steps at specific $B_{\text {ext }}$ values. We show that these features are a consequence of DW tilting due to magnetostatic effects, breaking the symmetry between the two ends of the DW.

The paper is organized as follows: In Sec. II we go through the details of our micromagnetic simulations, while in Sec. III we present our results, focusing on the multistep nature of the Walker breakdown in wide strips. Section IV finishes the paper with conclusions.

\section{SIMULATIONS}

Our micromagnetic simulations are performed using the GPU-accelerated micromagnetic simulation program $\mathrm{Mu}-$ Max3 [15]. It solves the space and time-dependent reduced magnetization $\mathbf{m}(\mathbf{r}, t)=\mathbf{M}(\mathbf{r}, t) / M_{\mathrm{s}}$ [with $\mathbf{M}(\mathbf{r}, t)$ and $M_{\mathrm{s}}$ the magnetization and saturation magnetization, respectively] 


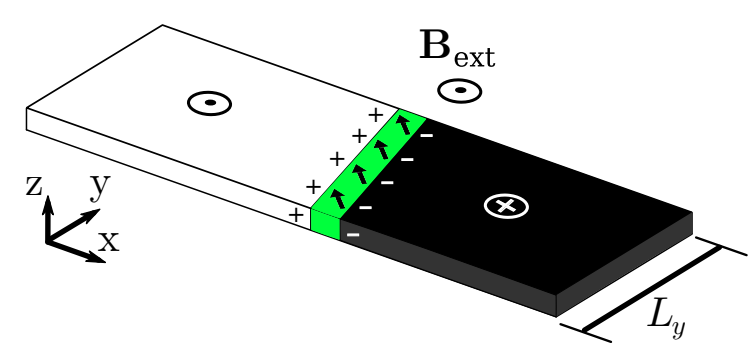

FIG. 1. Schematic representation of the simulated system. Two out-of-plane polarized domains are separated by a DW, which in equilibrium is a pure Bloch wall. As illustrated in the figure, upon application of an out-of-plane magnetic field $B_{\mathrm{ext}}<B_{\mathrm{W}}$, the magnetization of the moving DW finds a steady-state orientation corresponding to a partial Néel wall structure (arrows), producing magnetic charges on the DW surfaces. To minimize the resulting magnetostatic energy, the DW tries to orient itself with the DW magnetization, leading to DW tilting.

from the Landau-Lifshitz-Gilbert (LLG) equation,

$$
\frac{\partial \mathbf{m}}{\partial t}=-\frac{\gamma}{1+\alpha^{2}}\left\{\mathbf{m} \times \mathbf{B}_{\mathrm{eff}}+\alpha\left[\mathbf{m} \times\left(\mathbf{m} \times \mathbf{B}_{\mathrm{eff}}\right)\right]\right\},
$$

using a finite-difference discretization. In Eq. (1), $\gamma$ is the gyromagnetic ratio, $\alpha$ is the dimensionless damping parameter, and $\mathbf{B}_{\text {eff }}$ is the effective field having contributions from the externally applied field $\mathbf{B}_{\text {ext }}$, magnetostatic field, Heisenberg exchange field, as well as the anisotropy field. As a test system, we consider CoPtCr strips of thickness $L_{z}=12 \mathrm{~nm}$ and widths $L_{y}$ ranging from 90 to $1800 \mathrm{~nm}$. The length of the moving simulation window centered around the DW (implying that the dipolar fields due to the two domains cancel at the domain wall) is $L_{x}=3072 \mathrm{~nm}$. The system is discretized using cubic discretization cells with a side length of $3 \mathrm{~nm}$. The typical material parameters of $\mathrm{CoPtCr}[9,16]$ used here are uniaxial magnetic anisotropy $K_{\mathrm{u}}=2 \times 10^{5} \mathrm{~J} / \mathrm{m}^{3}$, exchange constant $A_{\mathrm{ex}}=10^{-11} \mathrm{~J} / \mathrm{m}$, damping parameter $\alpha=0.2$, and saturation magnetization $M_{\mathrm{s}}=3 \times 10^{5} \mathrm{~A} / \mathrm{m}$, corresponding to the stray field energy constant of $K_{\mathrm{d}}=\mu_{0} M_{\mathrm{s}}^{2} / 2=5.65 \times$ $10^{4} \mathrm{~J} / \mathrm{m}^{3}$, where $\mu_{0}$ is the vacuum permeability. These values result in the Bloch wall width parameter $\Delta=\sqrt{A_{\mathrm{ex}} / K_{\mathrm{u}}} \approx$ $7.1 \mathrm{~nm}$ and the Bloch line width parameter (or the exchange length) $\Lambda=\sqrt{A_{\mathrm{ex}} / K_{\mathrm{d}}} \approx 13.3 \mathrm{~nm}$.

The system is initialized in a configuration with two antiparallel out-of-plane $( \pm z)$ domains separated by a straight Bloch DW with the DW internal magnetization in the positive $y$ direction. The DW spans the strip width along the $y$ direction and is located in the middle of the sample. Upon sharp application of an external magnetic field $B_{\text {ext }}$ along the positive $z$ direction, the DW is displaced in the positive $x$ direction. The steady-state time-averaged DW velocities are then estimated from the slopes of the DW position vs time graphs, averaging over several cycles of the precessional DW dynamics for $B_{\text {ext }}>B_{\mathrm{W}}$ and excluding any initial transients.

At this point we note a crucial feature of field-driven DW dynamics in the strip geometry, illustrated in Fig. 1: A $B_{\text {ext }}$ smaller than the Walker field $B_{\mathrm{W}}$ tends to rotate the DW magnetization counterclockwise away from the positive $y$ direction (i.e., away from a pure Bloch wall configura-
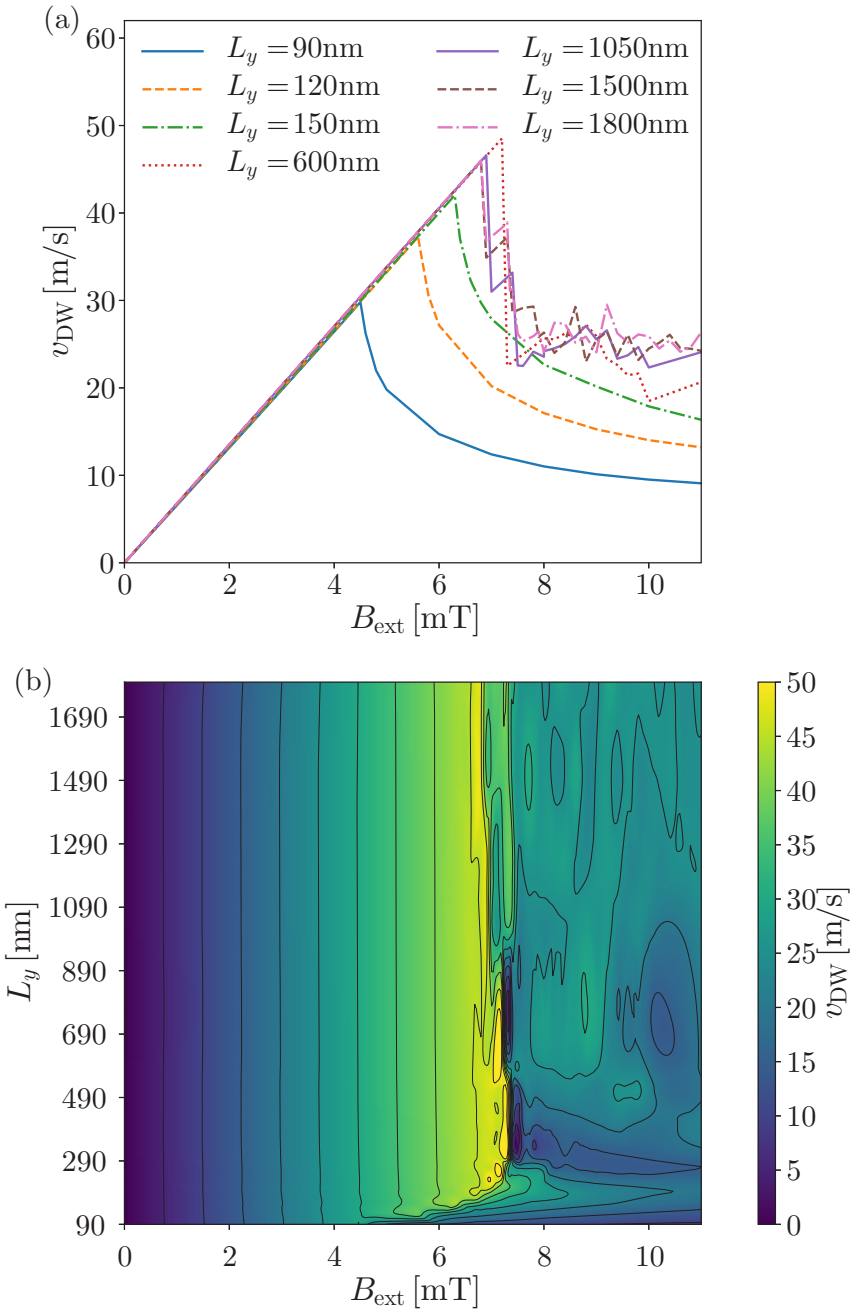

FIG. 2. (a) $v_{\mathrm{DW}}$ as a function of $B_{\text {ext }}$ considering a representative subset of different $L_{y}$ 's. Note the "smooth" velocity drop for narrow strips that changes first to a single large $\operatorname{drop}\left(L_{y}=600 \mathrm{~nm}\right)$ and then develops two or even three distinct velocity drops separated by short linear parts of the $v_{\mathrm{DW}}\left(B_{\text {ext }}\right)$ curve upon increasing $L_{y}$. (b) All the simulated $v_{\mathrm{DW}}\left(B_{\text {ext }}\right)$ data visualized as a contour plot, highlighting the nonmonotonic dependence of $B_{\mathrm{W}}$ on $L_{y}$.

tion), such that the moving steady-state DW acquires a Néel component (a finite $x$ component of the DW magnetization). This results in magnetic charges on the DW surfaces, with an associated cost in demagnetization energy. To minimize this energy, the DW tends to tilt in an attempt to align itself with the DW magnetization. A balance between the DW energy (proportional to the DW length) and the magnetostatic energy leads to a finite steady-state DW tilt angle (see Fig. 1). This mechanism will be crucial for understanding the properties of the Walker breakdown in the case of wide strips, discussed later in this paper.

\section{RESULTS}

We start by considering the relation between DW propagation velocity $v_{\mathrm{DW}}$ and $B_{\text {ext }}$ for strips of different widths. Figure 2(a) shows examples of $v_{\mathrm{DW}}\left(B_{\text {ext }}\right)$ curves, illustrating the key aspects of the observed DW dynamics. For all strip 

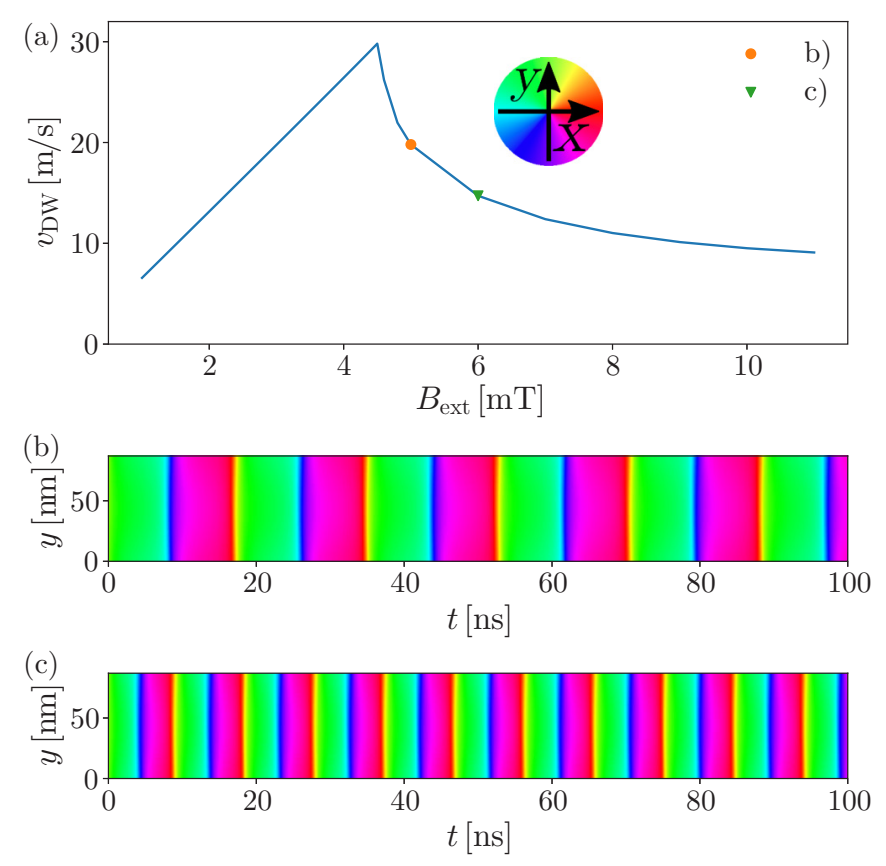

FIG. 3. (a) An example of a typical $v_{\text {DW }}\left(B_{\text {ext }}\right)$ curve of narrow strips (here, the $L_{y}=90 \mathrm{~nm}$ case is shown), exhibiting a smooth velocity drop for $B_{\mathrm{ext}}>B_{\mathrm{W}}$. Panels (b) and (c) display space-time maps of the internal DW magnetization (with the color wheel indicating the mapping from colors to magnetization) for two $B_{\text {ext }}$ values (5 and $6 \mathrm{mT}$, respectively) as indicated in (a) with the two symbols. These describe the time evolution of the internal in-plane magnetization of the domain wall for different $y$ coordinates along the domain wall. The in-plane DW magnetization exhibits coherent (spatially uniform) periodic switching events, with the frequency of the events increasing with $B_{\text {ext }}$.

widths the usual linear dependence of $v_{\mathrm{DW}}$ on $B_{\mathrm{ext}}$ for small $B_{\text {ext }}$ is terminated at an $L_{y}$-dependent Walker field $B_{\mathrm{W}}$. This is also depicted in the contour plot shown in Fig. 2(b). $B_{\mathrm{W}}$ first increases rapidly with $L_{y}$, reaches a maximum for $L_{y} \approx$ $350 \mathrm{~nm}$, after which $B_{\mathrm{W}}$ slowly decreases, possibly reaching a plateau for the largest $L_{y}$ values considered. This nonmonotonic $L_{y}$ dependence is reminiscent of our recent results on thickness-dependent Walker breakdown in garnet strips [10], and will be analyzed further below.

The shape of the $v_{\mathrm{DW}}\left(B_{\mathrm{ext}}\right)$ curve displaying the velocity drop crucially depends on $L_{y}$. For small $L_{y}$, corresponding to the regime where $B_{\mathrm{W}}\left(L_{y}\right)$ increases with $L_{y}$ [Fig. 2(b)], $v_{\text {DW }}$ decreases smoothly and gradually with increasing $B_{\text {ext }}$ [Figs. 2(a) and 3(a)]. Figures 3(b) and 3(c) display space-time maps of the DW internal in-plane magnetization during the dynamics; for each $y$ coordinate along the DW the magnetization shown is that of the midpoint of the DW where $m_{z}$ changes sign when moving along the $x$ direction. These maps show that above $B_{\mathrm{W}}$ the internal dynamics within the DW display the typical periodic switching of the DW magnetization [17], with the frequency of the switching events increasing with $B_{\text {ext }}$. Notably, for the rather narrow system with $L_{y}=90 \mathrm{~nm}$ (i.e., not much wider than the BL width $\pi \Lambda \approx 42 \mathrm{~nm}$ ) studied in Fig. 3, these switching events are to a very good approximation spatially uniform, such that the magnetization of the entire DW rotates synchronously, and no VBLs are observed.

This is in strong contrast to the behavior in wider strips $\left[L_{y} \gg \pi \Lambda\right.$ and beyond the maximum of $\left.B_{\mathrm{W}}\left(L_{y}\right)\right]$ : First, when increasing $L_{y}$, a single, quite steep velocity drop is observed; an example is given by the $L_{y}=600 \mathrm{~nm}$ curve in Fig. 2(a). For even wider strips, a remarkable feature is observed: Our simulations where we consider a finer sampling of the $B_{\text {ext }}$ values than previous studies [9] reveal that the Walker breakdown actually consists of multiple distinct velocity drops, separated by short linear parts of the $v_{\mathrm{DW}}\left(B_{\mathrm{ext}}\right)$ curve. First, for $L_{y}=1050 \mathrm{~nm}$ [Fig. 2(a)], we observe two velocity drops, and further increasing $L_{y}$ to $1500 \mathrm{~nm}$ leads to the appearance of three of these steps. All velocity drops take place within a rather narrow field range of less than $1 \mathrm{mT}$ (they all occur between 6.9 and $7.9 \mathrm{mT}$ ). Thus, they were not clearly observed in previous work [9], where the sampling of the $B_{\text {ext }}$ values was much more coarse.

To account for these distinct velocity drops, it is again instructive to consider the details of the underlying DW magnetization dynamics. Figure 4(a) shows an example of a $v_{\text {DW }}\left(B_{\text {ext }}\right)$ curve exhibiting three velocity drops, followed by a more irregular structure for larger $B_{\mathrm{ext}}\left(L_{y}=1500 \mathrm{~nm}\right)$. Subsequent to the first velocity drop (for $B_{\text {ext }} \approx 6.9 \mathrm{mT}$ ), as illustrated in the space-time map of DW internal magnetization in Fig. 4(b), a single VBL nucleates from the bottom edge of the strip, propagates along the DW across the strip width, exits the strip, after which another VBL of opposite $x$ magnetization [shown in red instead of blue in Fig. 4(b)] enters the strip/DW and propagates to the opposite strip edge, before the process repeats. Upon increasing $B_{\text {ext }}$ to $B_{\text {ext }} \approx$ $7.4 \mathrm{mT}$, a second velocity drop occurs. Figure 4(c) shows that this second drop is due to more complex VBL dynamics within the DW: After an initial transient, the system finds a steady state where another VBL is nucleated from the top strip edge before the VBL nucleated from the bottom edge reaches the top edge. Subsequently, the two VBLs annihilate within the strip, and a new pair of VBLs is created in the same DW segment. These two VBLs then propagate towards the bottom and top edges of the strip, respectively, and exit the strip. Thereafter, the process is repeated. A third velocity drop is observed for $B_{\text {ext }} \approx 7.9 \mathrm{mT}$, with the corresponding DW magnetization dynamics shown in Fig. 4(d): In this case, three VBLs are present within the DW for most of the time. Upon further increasing $B_{\text {ext }}$, the VBL dynamics become increasingly complex (not shown) and no further clear, distinct velocity drops can be resolved [Fig. 4(a)]. Movies illustrating the DW dynamics shown in Figs. 4(b)-4(d) are included as Supplemental Material [18]. Notice that while Figs. 4(b)4(d) describe the VBL dynamics along the DWs, the movies show in addition that DWs containing VBLs are not straight lines but tend to exhibit significant curvature especially at the locations of the VBLs.

The described dynamics of VBLs responsible for the distinct velocity drops crucially depend on a broken symmetry between the two ends of the DW (bottom vs top strip edges). As illustrated in Fig. 1, for $B_{\text {ext }}<B_{\mathrm{W}}$, the driving field rotates the magnetization of the moving DW away from a pure Bloch wall configuration to a steady DW structure with a finite Néel component. The Néel nature of the DW gives rise to magnetic 

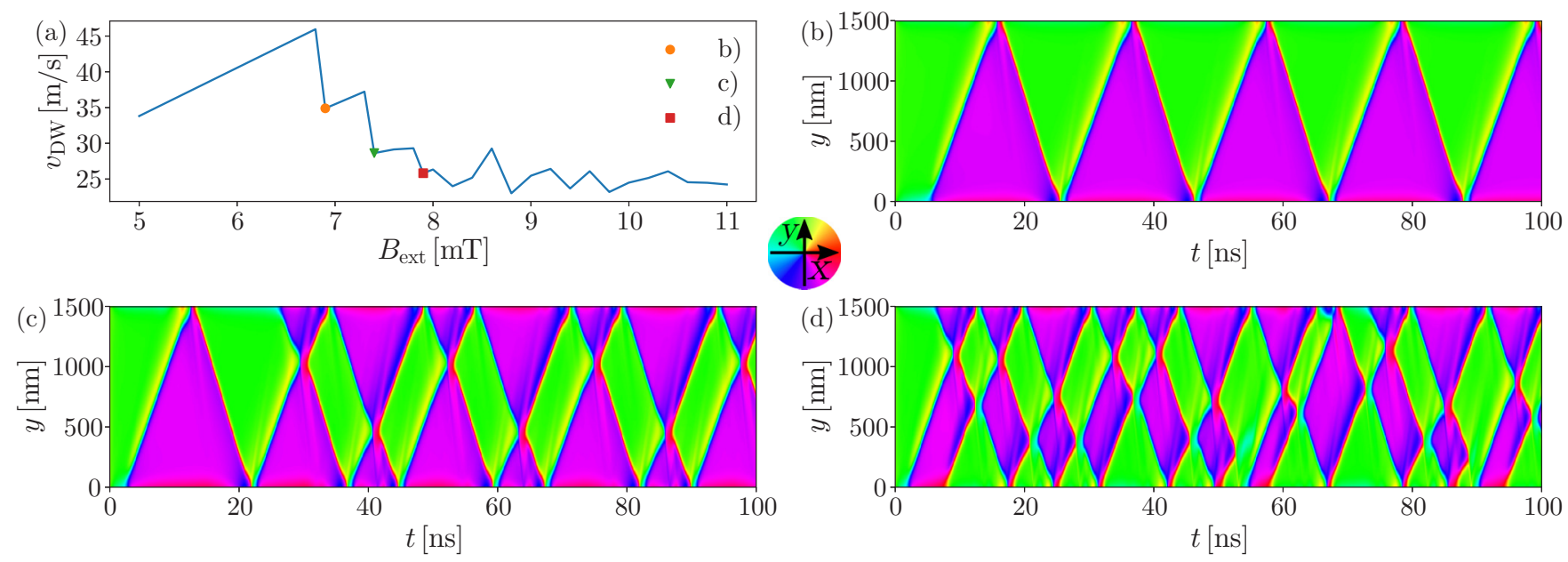

FIG. 4. A closer look at the DW dynamics corresponding to a multistep Walker breakdown for a strip of width $L_{y}=1500 \mathrm{~nm}$. The $v_{\text {DW }}\left(B_{\text {ext }}\right)$ curve shown in (a) exhibits three distinct velocity drops, with the corresponding VBL dynamics illustrated by means of space-time maps of the DW internal in-plane magnetization (with the color wheel in the middle showing the mapping from colors to magnetization direction) in (b)-(d). Panel (b) shows a single VBL first nucleating from the bottom edge (i.e., the leading end of the DW just before the onset of Walker breakdown), and then traveling back and forth along the DW. These dynamics are responsible for the first velocity drop seen in (a). Panel (c) displays the VBL dynamics corresponding to the second velocity drop, where after an initial transient a VBL is first nucleated from the bottom edge, and shortly afterwards a second VBL is nucleated from the top edge. Their annihilation is followed by an almost immediate formation of another pair of VBLs that propagate to the edges, after which the process repeats. Panel (d) shows the dynamics corresponding to the third velocity drop, involving the simultaneous presence of three VBLs within the DW. Movies illustrating the dynamics shown in (b)-(d) are included as Supplemental Material [18].

charges at the DW surfaces (Fig. 1). To reduce the resulting energy, the DW develops a tilt as it attempts to minimize the charges by aligning with its internal magnetization. Thus, the leading end of the DW effectively experiences a larger driving force (sum of $B_{\text {ext }}$ and the demagnetizing fields due to the DW surface charges) than the trailing one. Hence, when increasing $B_{\text {ext }}$ over the Walker threshold, the leading end of the DW experiences the breakdown first, i.e., at a lower $B_{\text {ext }}$, while the trailing end is still below its (local) Walker breakdown field. This means that the first VBL is always nucleated from the leading end of the DW [bottom edge in Figs. 4(b)-4(d)], and that the first velocity drop corresponds to a single VBL moving back and forth along the DW [Fig. 4(b)].

When $B_{\text {ext }}$ is increased to reach the second velocity drop, also the trailing end of the DW exceeds its local Walker threshold, and VBLs are nucleated from both ends of the DW. The leading end of the DW still experiences a larger effective driving force, and hence, the first VBL is nucleated from this edge. However, before it reaches the other end of the DW, a second VBL is nucleated from the trailing end, and subsequently the two Bloch lines annihilate inside the strip, followed by creation of a new pair of VBLs in the same location [Fig. 4(c)]. Increasing $B_{\text {ext }}$ even more to reach the third velocity drop leads to nucleation of a third VBL, while the two first ones are still inside the strip, resulting in the simultaneous presence of three VBLs along the DW [Fig. 4(d)]. We note that all creation and annihilation reactions in Fig. 4 respect the conservation of the magnetic charge $Q= \pm 1$ and chirality $C= \pm 1 / 2$ of the fourfold degenerate VBLs [19].

Finally, we address the nonmonotonic dependence of the Walker field $B_{\mathrm{W}}$ (defined as the $B_{\text {ext }}$ value where the first velocity drop takes place) on $L_{y}$ (see Fig. 2). As found by Mougin et al. [8], in confined geometries with uniform magnetization along the DW $B_{\mathrm{W}} \propto\left|N_{x}-N_{y}\right|$, where $N_{x}$ and $N_{y}$ denote the demagnetizing factors of the DW along $x$ and $y$, respectively. Employing the elliptic approximation leads to $N_{x} \approx L_{z} /\left(L_{z}+\pi \Delta\right)$ and $N_{y} \approx L_{z} /\left(L_{z}+L_{y}\right)[8,20]$. Notice that the DW width $\pi \Delta \approx 22.3 \mathrm{~nm}$ used above can be obtained by integrating the Bloch wall profile $m_{y}=1 / \cosh (x / \Delta)$ [21], and the approximate expressions for $N_{x}$ and $N_{y}$ utilized are valid for $L_{z} \ll \pi \Delta$ and $L_{z} \ll L_{y}$, respectively. Thus, we obtain the approximate result that $B_{\mathrm{W}} \propto \mid L_{z} /\left(L_{z}+\pi \Delta\right)-L_{z} /\left(L_{z}+\right.$ $\left.L_{y}\right) \mid$, suggesting that $B_{\mathrm{W}}$ increases with $L_{y}$, in agreement with our observations for narrow strips (small $L_{y}$ ), where the magnetization of the entire DW precesses in phase above the breakdown (see Fig. 3). However, the above expression also predicts a saturation of $B_{\mathrm{W}}$ in the limit $L_{y} \gg L_{z}$, at odds with our observation in Fig. 2 where, after reaching a maximum, $B_{\mathrm{W}}$ is slowly decreasing with $L_{y}$. Indeed, the calculation in [8] is valid for uniform DW magnetization only. In particular, it does not take into account the possibility of nucleation of VBLs within the DW which is the mechanism underlying the Walker breakdown for large $L_{y}$. The energy barrier for VBL nucleation should depend on $L_{y}$, such that it is lower for longer DWs (larger $L_{y}$ ). However, for the very largest strip widths $L_{y}$ considered (1500 and $1800 \mathrm{~nm}), B_{\mathrm{W}}$ appears to saturate to a value of $B_{\mathrm{W}} \approx 6.7 \mathrm{mT}$.

\section{CONCLUSIONS}

Thus, we have established that precessional DW dynamics in PMA strips undergo a transition from spatially homogeneous precession of the DW magnetization to a VBLdominated regime as the strip width $L_{y}$ is increased. The latter 
regime is characterized by multiple distinct velocity drops in the $v_{\text {DW }}\left(B_{\text {ext }}\right)$ curve, originating from asymmetric nucleation of VBLs from the strip edges due to DW tilting. This closer look at the well-studied phenomenon of Walker breakdown thus reveals its multistep nature for DWs with lengths well above the VBL width. These features should lend themselves to experimental verification in future studies. It would also be of interest to extend our study to systems with structural disorder or inhomogeneities interacting with the DW [22,23], to consider the possible effects of a small tilt of the applied field, as well as to investigate other materials characterized by different micromagnetic parameters; considering such details numerically would be helpful in better understanding the experimental conditions where the mechanism reported here could be observed. We would expect that the multistep nature of Walker breakdown should be experimentally observable whenever the disorder-induced depinning field is well below the Walker field. Another future avenue of research of considerable current interest would be to address the effect of a finite Dzyaloshinskii-Moriya interaction (DMI) [24], resulting in a scenario where the degeneracy of the different VBL configurations is lifted due to DMI-induced splitting of the energy levels [19].

\section{ACKNOWLEDGMENTS}

This work has been supported by the Academy of Finland through an Academy Research Fellowship (L.L.; Project No. 268302). We acknowledge the computational resources provided by the Aalto University School of Science "Science-IT" project, as well as those provided by CSC (Finland).
[1] G. S. Beach, C. Nistor, C. Knutson, M. Tsoi, and J. L. Erskine, Nat. Mater. 4, 741 (2005).

[2] P. J. Metaxas, J. P. Jamet, A. Mougin, M. Cormier, J. Ferré, V. Baltz, B. Rodmacq, B. Dieny, and R. L. Stamps, Phys. Rev. Lett. 99, 217208 (2007).

[3] N. L. Schryer and L. R. Walker, J. Appl. Phys. 45, 5406 (1974).

[4] S. S. Parkin, M. Hayashi, and L. Thomas, Science 320, 190 (2008).

[5] A. Thiaville, Y. Nakatani, J. Miltat, and Y. Suzuki, Europhys. Lett. 69, 990 (2005).

[6] T. A. Moore, I. Miron, G. Gaudin, G. Serret, S. Auffret, B. Rodmacq, A. Schuhl, S. Pizzini, J. Vogel, and M. Bonfim, Appl. Phys. Lett. 93, 262504 (2008).

[7] A. Thiaville and Y. Nakatani, in Spin Dynamics in Confined Magnetic Structures III (Springer, New York, 2006), pp. 161-205.

[8] A. Mougin, M. Cormier, J. Adam, P. Metaxas, and J. Ferré, Europhys. Lett. 78, 57007 (2007).

[9] T. Herranen and L. Laurson, Phys. Rev. B 92, 100405(R) (2015).

[10] T. Herranen and L. Laurson, Phys. Rev. B 96, 144422 (2017).

[11] A. Thiaville and J. Miltat, in Topology in Magnetism (Springer, New York, 2018), pp. 41-73.

[12] D. A. Garanin, E. M. Chudnovsky, and X. Zhang, Europhys. Lett. 120, 17005 (2017).

[13] A. Malozemoff and J. Slonczewski, Magnetic Domain Walls in Bubble Materials: Advances in Materials and Device Research (Academic, New York, 1979), Vol. 1.
[14] S. Konishi, IEEE Trans. Magn. 19, 1838 (1983).

[15] A. Vansteenkiste, J. Leliaert, M. Dvornik, M. Helsen, F. GarciaSanchez, and B. Van Waeyenberge, AIP Adv. 4, 107133 (2014).

[16] D. Weller, A. Moser, L. Folks, M. E. Best, W. Lee, M. F. Toney, M. Schwickert, J.-U. Thiele, and M. F. Doerner, IEEE Trans. Magn. 36, 10 (2000).

[17] E. Martinez, J. Phys.: Condens. Matter 24, 024206 (2011).

[18] See Supplemental Material at http://link.aps.org/supplemental/ 10.1103/PhysRevB.99.174427 for movies illustrating the DW dynamics responsible for the three different velocity drops.

[19] Y. Yoshimura, K.-J. Kim, T. Taniguchi, T. Tono, K. Ueda, R. Hiramatsu, T. Moriyama, K. Yamada, Y. Nakatani, and T. Ono, Nat. Phys. 12, 157 (2016).

[20] O. Boulle, G. Malinowski, and M. Kläui, Mater. Sci. Eng. R Rep. 72, 159 (2011).

[21] A. Hubert and R. Schäfer, Magnetic Domains: The Analysis of Magnetic Microstructures (Springer Science \& Business Media, New York, 2008).

[22] J. Leliaert, B. Van de Wiele, A. Vansteenkiste, L. Laurson, G. Durin, L. Dupré, and B. Van Waeyenberge, J. Appl. Phys. 115, 17D102 (2014).

[23] J. Leliaert, B. Van de Wiele, A. Vansteenkiste, L. Laurson, G. Durin, L. Dupré, and B. Van Waeyenberge, J. Appl. Phys. 115, 233903 (2014).

[24] A. Thiaville, S. Rohart, É. Jué, V. Cros, and A. Fert, Europhys. Lett. 100, 57002 (2012). 